Onkologe 2022 $28: 340-346$

https://doi.org/10.1007/s00761-022-01102-1

Angenommen: 20. Januar 2022

Online publiziert: 17. Februar 2022

(c) The Author(s), under exclusive licence to Springer Medizin Verlag GmbH, ein Teil von Springer Nature 2022

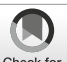

\section{Post-Corona-Fatigue - das bekannte Bild in neuem Gewand?}

\author{
Barbara Buchberger ${ }^{1,2} \cdot$ Ruben Zwierlein ${ }^{3} \cdot$ Volker Rohde $^{3,4}$ \\ ${ }^{1}$ Robert Koch-Institut, Berlin, Deutschland \\ ${ }^{2}$ Lehrstuhl für Medizinmanagement, Universität Duisburg-Essen, Essen, Deutschland \\ ${ }^{3}$ Helios Agnes Karll Krankenhaus, Bad Schwartau, Deutschland \\ ${ }^{4}$ Praxis für Urologie, Bad Schwartau, Deutschland
}

\title{
Zusammenfassung
}

Hintergrund: Die myalgische Enzephalitis oder das "chronic fatigue syndrome“ (ME/CFS) ist als Folge von COVID(Corona Virus Disease)-19 erneut in den Fokus gerückt. Grundsätzlich problematisch ist die Tatsache, dass ME/CFS als eigenständige Entität gilt, aber extreme Fatigue auch ein häufiges Symptom einer zugrunde liegenden Erkrankung ist. Ziel des Beitrags ist es, die Akzeptanz für ME/CFS und extreme Fatigue bei nicht vollumfänglich verstandener Symptomatologie zu erhöhen und den Bedarf an Forschung, Orientierung für niedergelassene Ärzte sowie an Beratungsangeboten für Patienten hervorzuheben

Material und Methoden: Orientierende Recherchen in Form einer fokussierten Informationsbeschaffung.

Ergebnisse: In verschiedenen Forschungsprojekten konnte die Hypothese von postinfektiöser ME/CFS als Autoimmunerkrankung bestätigt werden. Generell erschweren sowohl die Heterogenität der diagnostischen Kriterien als auch die Vielfalt von Formulierungen zur Beschreibung der Symptomatik sowie verschiedene Codierungsmöglichkeiten eine klare Zuordnung der Symptome zu einem Krankheitsbild. Belastungsintoleranz wurde als schwerwiegendes Symptom einer Post-COVID-19-Erkrankung identifiziert. Aus diesem Grund werden aktuell die Empfehlungen in internationalen Leitlinien insbesondere hinsichtlich von Pacing überarbeitet. Die Auswirkungen auf Empfehlungen bei tumorbedingter Fatigue oder bei MS sind unklar.

Schlussfolgerung: Vor dem Hintergrund eines abnehmenden "burden of disease" durch steigende Impfraten sollte die Forschung zu Fatigue nicht nur viral bedingte Erkrankungen umfassen.

\section{Schlüsselwörter}

Myalgische Enzephalitis · "Chronic fatigue syndrome" · ME/CFS · "Post-exertional malaise" · PEM . Belastungsintoleranz $\cdot$ Pacing

\section{Hintergrund}

"Es ist, als würde jemand den Stecker ziehen, den Hauptschalter umlegen", beschreibt eine Betroffene ihren Zustand in der ARTE-Reportage "Die rätselhafte Krankheit - Leben mit ME/CFS", die am 10. Juli 2021 ausgestrahlt wurde [1].

Die myalgische Enzephalitis oder das "chronic fatigue syndrome" (ME/CFS) hat als Folge einer COVID(Corona Virus Disease)-19-Erkrankung neue Aufmerksamkeit erhalten. Allein in Deutschland sind ca. 300.000 Menschen betroffen, und durch die SARS-CoV-2-Pandemie steigt diese Zahl weiter an [2]. In einem systematischen Review fanden Nasserie et al. in 25 von 45 eingeschlossenen Studien nach Kurzatmigkeit und Dyspnoe Fatigue als zweithäufigstes persistierendes Symptom von Long-COVID mit einer medianen Häufigkeit von 40,0\% (IQR: 31,0-57,0\%) [3].

Fatigue kann als ein durchdringendes Gefühl von Müdigkeit oder Energielosigkeit definiert werden, das nicht ausschließlich auf Anstrengung zurückzuführen ist. Sie ist in den meisten Fällen nur vorübergehend und von Muskelermüdung oder -schwäche zu unterscheiden. Wenn die Er- 
Tab. 1 Mögliche Ursachen abnormer Erschöpfung [5]

Akute oder chronische Infektion

\begin{tabular}{|c|c|}
\hline - Viral & - Parasitär \\
\hline - Bakteriell & - Zoonotisch \\
\hline \multicolumn{2}{|l|}{ Stimmungsschwankung } \\
\hline \multicolumn{2}{|c|}{ Schlafstörungen inklusive Schlafapnoe } \\
\hline \multicolumn{2}{|c|}{ Bösartige Neubildung jeglicher Art } \\
\hline \multicolumn{2}{|l|}{ Herz-Kreislauf-Erkrankung } \\
\hline - Agranulozytose & - Subakute bakterielle Endokarditis \\
\hline - Anämie & - Leukämie \\
\hline - Herzinsuffizienz & - Mitralstenose \\
\hline - Kongenitale Herzerkrankung & - Myokarditis \\
\hline - Cor pulmonale & \multirow{2}{*}{ - Thalassaemia major oder minor } \\
\hline - Endokarditis & \\
\hline \multicolumn{2}{|c|}{ Stoffwechselerkrankungen/-störungen } \\
\hline - Avitaminose & - Euthyroid-sick-Syndrom \\
\hline - Diabetes mellitus & - Menopause \\
\hline - Elektrolytstörungen & - Stoffwechselerkrankungen \\
\hline - Hyperparathyreoidismus & Phenylketonurie \\
\hline - Hypothyreose & Fanconi-Syndrom \\
\hline - Hyperthyreose & Zystische Fibrose \\
\hline \multicolumn{2}{|l|}{ Hormonerkrankungen/-störungen } \\
\hline - Akromegalie & \multirow{2}{*}{ - Hypopituitarismus } \\
\hline - Morbus Addison & \\
\hline \multicolumn{2}{|l|}{ Kollagenosen } \\
\hline - Rheumatoide Arthritis & \multirow[t]{2}{*}{ - Sklerodermie } \\
\hline - Lupus erythematodes & \\
\hline \multicolumn{2}{|l|}{ Chronische Allergiesymptome } \\
\hline \multicolumn{2}{|c|}{ Schwermetallvergiftung (Bsp.: Blei, Quecksilber, Arsen) } \\
\hline \multicolumn{2}{|c|}{ Andere } \\
\hline - ME/CFS & - Multiple Sklerose \\
\hline - Cushing-Syndrom & - Myasthenia gravis \\
\hline - Myelodysplastisches Syndrom & - Myelofibrose \\
\hline - Sick-building-Syndrom & - Osteomalazie und Rachitis \\
\hline - Postpoliosyndrom & - Parkinson-Krankheit \\
\hline - Arteriitis temporalis & - Morbus Vazquez-Osler \\
\hline - Ulzerative Kolitis & - Polymyalgia rheumatica \\
\hline - Malabsorptionssyndrom & - Polyneuritis \\
\hline - Hodgkin-Lymphom & - Rheumatisches Fieber \\
\hline - Schlaganfall & \multirow[t]{2}{*}{ - Zirrhose/Urämie } \\
\hline - Kopfverletzung & \\
\hline
\end{tabular}

schöpfung jedoch länger andauert oder zu schweren Beeinträchtigungen wie zum Beispiel Aufmerksamkeits- und Konzentrationsstörungen, die eine berufliche Tätigkeit unmöglich machen, führt und von anderen charakteristischen konstitutionellen und neuropsychiatrischen Symptomen begleitet wird, sollte die Diagnose eines chronischen Fatiguesyndroms (CFS) in Betracht gezogen werden $[4,5]$.
Mehrere klinische Hauptprobleme sind mit der Diagnose von abnormalen Müdigkeitszuständen verbunden. Das grundsätzliche diagnostische Problem ist die Bestimmung der Ursache der Erschöpfung. Zu beachten ist, dass ME/CFS eine eigenständige Entität ist, aber extreme Fatigue auch ein häufiges Symptom einer zugrunde liegenden Erkrankung wie beispielsweise einer Tumorerkrankung oder multiplen Sklerose (MS). Ein gewisser Anteil von
Patienten mit schwerer tumorassoziierter Fatigue oder Fatigue als Symptom bei MS erfüllt die kanadischen Konsensuskriterien für ME/CFS. Diese Symptome können als "CFS-like" bezeichnet werden (siehe unten und - Tab. 1; [5]). Erschwerend kommen für die Diagnostik ein weites Spektrum an Formulierungen zur Beschreibung der Symptomatik, eine große Spannbreite von Beschwerden [6] sowie Verzerrungen durch Übersetzungen aus dem Englischen hinzu.

Ziel des vorliegenden Beitrags ist es, die Akzeptanz für ME/CFS und extreme Fatigue bei nicht vollumfänglich verstandener Symptomatologie zu erhöhen. Darüber hinaus wird der Bedarf an Forschung, Orientierung für niedergelassene Ärzte sowie an Beratungsangeboten für Patienten hervorgehoben.

\section{Methoden}

Es wurde eine orientierende Recherche in Form einer fokussierten Informationsbeschaffung der für die Fragestellung angemessenen aktuellen Literatur durchgeführt.

\section{$\mathrm{ME} / \mathrm{CFS}$ in Folge von COVID- 19 verglichen mit anderen Krankheitsbildern}

Im Vergleich mit der als Pandemie erwarteten Influenza ergaben Analysen der Grippedatenbanken der WHO bereits zu Beginn der Maßnahmen zum "containment "von SARS-CoV-2 eine deutlich höhere Übertragbarkeit des Erregers [7]. Auch für das Krankheitsbild COVID-19 ließen retrospektive Kohortenstudien, wie die von Benaim et al. mit Daten aus einer Einrichtung der Maximalversorgung in Israel, auf ein erheblich komplexeres Krankheitsbild schließen. Die Auswirkungen von COVID19 auf mehrere kritische Funktionen wie Immunsystem, Stoffwechsel, Blutgerinnung sowie Herz- und Atmungsfunktion stellten sich partiell als gravierend heraus [8]. Das multiorganische Erscheinungsbild sowie thromboembolische Ereignisse lassen darauf schließen, dass SARS-CoV-2 ein endotheliophiles Virus ist und COVID19 daher auch hinsichtlich der Spätfolgen eher mit einer Sepsis als mit einer Grippe vergleichbar ist [9]. Weitere Un- 


\section{Infobox 1}

IOM-Kriterien [15]

Vorgeschlagene diagnostische Kriterien für $\mathrm{ME} / \mathrm{CFS}$.

Die folgenden 3 Kriterien müssen erfüllt sein:

1. Eine erhebliche Reduktion oder

Beeinträchtigung bei der Ausübung von

Beruf, Bildung, sozialen oder persönlichen

Aktivitäten,

- die mehr als 6 Monate anhält und von (oft stark ausgeprägter) Erschöpfung begleitet ist

- die einen eindeutigen Beginn aufweist (nicht von Geburt an)

- die nicht das Resultat von fortwährender und anhaltender Belastung ist

- die sich durch Schonung und Ausruhen nicht erheblich lindern lässt

2. Zustandsverschlechterung nach

Anstrengung (PEM ${ }^{\mathrm{a}}$ )

4. Mindestens eine der folgenden

Beschwerden muss vorliegen:

- Kognitive Beeinträchtigung

- Orthostatische Intoleranz

aHäufigkeit und Schweregrad der Symptome sollten festgestellt werden.

Die Diagnose ME/CFS (SEID) sollte hinterfragt werden, wenn Patienten diese Symptome mindestens die Hälfte der Zeit nicht mit moderater, bedeutender oder schwerer Intensität aufweisen
3. Nicht erholsamer Schlaf

\section{Infobox 2}

Die kanadischen Konsensuskriterien für die Diagnose ME/CFS [6]

Die nachstehend aufgeführten Symptome müssen seit mindestens sechs Monaten bei Erwachsenen (drei Monate bei Kindern und Jugendlichen) anhaltend oder wiederkehrend auftreten.

Liegen andere Erkrankungen mit den gleichen Symptome vor, müssen diese Erkrankungen zuerst untersucht und optimal behandelt werden, bevor die Diagnose ME/CFS gestellt werden kann. Auszuschließende Erkrankungen sollten durch eine Kombination aus klinischer Anamnese, körperlicher Untersuchung und ergänzenden Tests abgeklärt werden.

- Pathologische Fatigue

- Zustandsverschlechterung nach Anstrengung (PEM)

- Schlafstörungen

- Schmerzen

- Kognitive Symptome (mindestens zwei Symptome aus einer vorgegebenen Liste)

Darüber hinaus ist mindestens ein Symptom aus zwei der folgenden Kategorien von Symptomen erforderlich:

- Autonom

- Neuroendokrin

- Das Immunsystem betreffend

tersuchungen ergaben, dass das SARSCoV-2-Spike-Protein die Bildung von Inflammasomen und die Freisetzung von Interleukinen anregt; darüber hinaus wurde eine tiefgreifende und lang anhaltende Umprogrammierung von Makrophagen beobachtet, was zu einer erhöhten Immunogenität des SARS-CoV-2-S-Proteins führt [10]. In Gewebeproben aus dem frontalen Kortex und Plexus choroideus wurden Perturbationen der zellulären Mechanismen beobachtet, aus denen sich ableiten lässt, dass die Entzündung in diesen Zellen an der Diffusionsbarriere registriert und dem Gehirn signalisiert wird, dass periphere T-Zellen das Parenchym infiltrieren und das Entzündungsgeschehen zusätzlich befeuern. Es fanden sich Subpopulationen von Astrozyten und Mikroglia, die pathologisch zelluläre Merkmale aufwiesen, die bei neurodegenerativen Erkrankungen auftreten [11]. Halpert und Shoenfeld konnten bereits 2020 in einer Subgruppe von Patienten Hinweise darauf finden, dass das Virus eine kritische und zentrale
Wirkung auf die menschliche Immunität hat und bei genetischer Prädisposition Autoimmunerkrankungen auslösen kann; sie bezeichneten SARS-CoV-2 daher als Autoimmunvirus [12]. Die Hypothese, dass SARS-CoV-2 die Bildung autoaggressiver Antikörper auslöst und daher zur Einschränkung der Hirnfunktion führt, bestätigten auch andere Forschergruppen wie die um den Neuropathologen Markus Glatzel vom UKE Hamburg. In der Postmortem-Fallserie von 43 Patienten fanden sie am häufigsten leichte neuropathologische Veränderungen mit ausgeprägter Neuroinflammation im Hirnstamm [13]. Die stellvertretende Direktorin des Instituts für Medizinische Immunologie an der Berliner Charité, Carmen Scheibenbogen, geht davon aus, dass es sich bei postinfektiöser ME/CFS um eine Autoimmunerkrankung handelt. In ihrer Untersuchung einer kleinen Subgruppe von jüngeren $\mathrm{Pa}$ tienten nach milder bis moderater COVID19-Infektion zeigte sich, dass COVID-19 ein schweres chronisches Syndrom aus- lösen kann, das sich durch Fatigue und Belastungsintoleranz auszeichnet [14].

\section{Heterogenität diagnostischer Kriterien für ME/CFS}

Die am häufigsten verwendeten diagnostischen Kriterienkataloge sind die Canadian Consensus Criteria für ME/CFS von 2003 und die Criteria for SEID (Systemic Exertion Intolerance Disease) des American Institute of Medicine (IOM) von 2015 [15]. Ebenfalls zur Anwendung kommen die Four-Symptom Criteria, die von Jason et al. als 1) Erschöpfung oder extreme Müdigkeit, 2) Schwierigkeiten bei der Wortfindung oder dem Ausdrücken von Gedanken, 3) körperliche Erschöpfung oder Krankheit nach leichter Aktivität und 4) nicht erholsamer Schlaf definiert wurden [16].

Mit den Kriterien werden unterschiedliche Patientenpopulationen beschrieben. Die Definitionen durch die Four-Symptom Criteria und die IOM-Kriterien für SEID (siehe - Infobox 1) umfassen kleinere Patientenpopulationen als die kanadischen Konsensuskriterien (siehe - Infobox 2). Letztere und die IOM-Kriterien beinhalten die Post-Exertional Malaise (PEM; [17]), die als zentrales Merkmal des Krankheitsbilds ME/CFS betrachtet wird und die Verschlechterung der Symptomatik nach körperlicher und kognitiver Anstrengung beschreibt [18].

\section{Empfehlungen zu körperlicher und kognitiver Anstrengung}

Bei tumorassoziierter Fatigue werden in den Leitlinien der europäischen Fachgesellschaft für medizinische Onkologie von 2020 körperliche Betätigung von moderater Intensität, Aerobic und Funktionstraining explizit empfohlen. Darüber hinaus wird zu Spazierengehen sowie Aerobic und Funktionstraining zu Hause geraten, um Fatigue und Lebensqualität zu verbessern [19]. In der Leitlinie wird beschrieben, dass sich die tumorassoziierte Fatigue von anderen Arten der Fatigue durch die Unfähigkeit, sie durch Ruhe oder Schlaf zu lindern, ihre Schwere und Persistenz unterscheidet. Sie betrifft fast $65 \%$ der Patienten mit einer Tumorerkrankung, und über zwei Drittel dieser Patienten beschreiben die tumor- 


\section{Infobox 3}

Websites mit weiterführenden Informationen

- Bundesverband ME/CFS Fatigatio e.V. Fatigatio e. V. - Bundesverband ME/CFS https://www.fatigatio.de/

- Deutsche Gesellschaft für ME/CFS, https:// www.mecfs.de/

- Lost Voices Stiftung Hilfe für Menschen mit ME/CFS, https://lost-voices-stiftung. org/

- Die Deutsche Fatigue Gesellschaft, https:// deutsche-fatigue-gesellschaft.de/

- Für Ärzte: Charité Fatigue Centrum, https://cfc.charite.de/fuer_aerzte/

- Euromene - Europäisches Netzwerk zu ME/CFS, https://www.cost.eu/actions/ CA15111/

assoziierte Fatigue als schwerwiegend für mindestens sechs Monate, und ein Drittel berichtet über eine für einige Jahre nach der Behandlung anhaltende Fatigue. Bis zu $40 \%$ der Patienten berichten über Fatigue bei der Krebsdiagnose, 80-90\% während der Chemotherapie und/oder Strahlentherapie. Darüber hinaus können Hormontherapie, zielgerichtete Therapie und auch Immuntherapie für die Fatigue verantwortlich sein. Im Zusammenhang mit einer Immuntherapie hat die tumorassoziierte Fatigue eine Inzidenz von 12 bis $37 \%$. Wenn die Immuntherapie mit Chemotherapie, monoklonalen Antikörpern, antiangiogenen Mitteln und zielgerichteten Therapien kombiniert wird, steigt die Inzidenz auf bis zu $71 \%$. Darüber hinaus kann eine Immuntherapie eine Ursache für Fatigue sein, wenn sie durch endokrinologische Störungen kompliziert wird [19].

Auch in klinischen Leitlinien zum Management von MS wird behandelnden Ärzten geraten, Betroffene darauf hinzuweisen, dass Aerobic, Gleichgewichtsund Dehnungsübungen einschließlich Yoga bei der Behandlung von MS-bedingter Fatigue hilfreich sein können [20]. In einer aktuellen norwegischen Studie mit 1600 Teilnehmern wurde eine Prävalenz für Fatigue bei MS-Patienten von $81 \%$ ermittelt und auf ältere Studien mit einer Prävalenz zwischen 50 und $90 \%$ verwiesen [21].

Im Gegensatz zu den Empfehlungen für tumorassoziierte Fatigue wird in der im Oktober 2021 veröffentlichten britischen Leitlinie des National Institute for Health and Care Excellence (NICE) zu Diagnose und Management von ME/CFS darauf hingewiesen, dass Programme für eine schrittweise Steigerung der körperlichen Aktivität oder des Trainings nicht zur Behandlung von ME/CFS angeboten werden sollten. Hintergrund sind die Schäden, die von Menschen mit ME/CFS berichtet wurden. Stattdessen wird betont, wie wichtig es sei, sicherzustellen, dass die Betroffenen bei Aktivitäten in ihrem „Energiebereich" bleiben. Zudem wird empfohlen, dass Programme für körperliche Aktivität für Menschen mit ME/CFS nur unter bestimmten Umständen in Betracht gezogen werden sollen [22]. Ein solches Aktivitätsmanagement wird auch als Pacing bezeichnet. Unter Pacing ist ein schonender Umgang mit individuellen Energiereserven im Alltag zu verstehen, sowohl auf körperlicher als auch auf kognitiver und emotionaler Ebene. Die Patienten werden dafür sensibilisiert, für sie typische Warnsignale unter sozialer, kognitiver, emotionaler und körperlicher Aktivität zu erkennen, zum Beispiel durch das Führen von Symptomtagebüchern [23]. Durch rechtzeitiges Unterbrechen oder Beenden einer Aktivität kann ein gefürchteter Zusammenbruch (Crash/PEM) vermieden werden. PEM ist nicht mit Ermüdungserscheinungen vergleichbar, die sich bei gesunden Menschen infolge von körperlicher Aktivität, Anstrengung oder Aufregung einstellen; sie kann sofort oder verzögert nach geringster Aktivität auftreten und bis zur Bettlägerigkeit führen. Oftmals sind viele Tage zur Erholung notwendig, und eine Besserung tritt nur langsam ein. Ihre persönlichen aktuellen Grenzen können Patienten leichter akzeptieren, wenn sie durch eine medizinische Vertrauensperson bei der Erfassung und Einordnung der Symptome unterstützt werden. Ein konsequent praktiziertes Pacing gibt Patienten mehr Sicherheit bei Aktivitäten jeglicher Art und kann eine Grundlage zur Verbesserung des physischen und psychischen Wohlbefindens bilden [22, 24].

In der NICE-Leitlinie, in der Evidenz zu ME/CFS infolge einer COVID-19-Erkrankung explizit nicht berücksichtigt ist, wird die Balance der IOM-Kriterien positiv bewertet, aber eine Anpassung gefordert. Kombination und Interaktion der vier Hauptkriterien grenzen ME/CFS von anderen Erkrankungen ab, weswegen alle vier Kriterien (lähmende Fatigue, PEM, nicht erholsamer Schlaf, Schlafstörungen oder beides und kognitive Schwierigkeiten) für eine Diagnose vorliegen sollten. In diesem Fall kann auch eine akute Erkrankung ausgeschlossen werden und die Anforderung an die Dauer des Anhaltens aller vier Symptome für die Diagnose ME/CFS auf mindestens sechs Wochen bei Erwachsenen und vier Wochen bei Kindern reduziert werden.

Betont wird darüber hinaus, dass die kognitive Verhaltenstherapie (CBT) keine Behandlung oder Heilmittel für ME/CFS sein kann. CBT kann als unterstützende Therapie zur Symptombewältigung und Verbesserung von Wohlbefinden und Lebensqualität geeignet sein [22].

\section{Diskussion}

In ihrer Stellungnahme kam die Deutsche Gesellschaft für ME/CFS e.V. unter Berufung auf den IOM-Report und den systematischen Review von Hvidberg et al. bereits im Jahr 2017 zu dem Schluss, dass die Schwere der Erkrankung, die im Durchschnitt sehr niedrige Lebensqualität, die Prävalenz sowie eine hohe Dunkelziffer von bis zu $90 \%$ es dringend notwendig machen, das Krankheitsbild ME/CFS in der Fachöffentlichkeit bekannter zu machen $[15,25,26]$. Ihrer Forderung nach einem massiven Ausbau der Versorgung und Intensivierung der Forschungsanstrengungen hat die SARS-CoV-2-Pandemie mit ME/CFS infolge einer COVID-19-Infektion deutlichen Vorschub geleistet. Kedor et al. gehen davon aus, dass sich die Zahl der ME/CFS-Patienten durch die Pandemie dramatisch erhöhen könnte [14].

Nicht nur aufgrund der Tatsache, dass auch Patienten mit z. B. tumorassoziierter Fatigue die kanadischen Konsensuskriterien für ME/CFS erfüllen können, ist es wünschenswert, dass Forschungsaktivitäten nicht nur auf viral bedingte Fatigue fokussiert werden. Angesichts der hohen Prävalenz und Inzidenz von Krebserkrankungen und MS stellt sich auch die Frage, wie groß der nationale und internationale „burden of disease" von postviraler Fatigue infolge einer COVID-19-Erkrankung tatsächlich ist und wie er sich angesichts der Impfquoten entwickeln wird. Die aktu- 
Hier steht eine Anzeige.

黑 Springer 
ellen Forschungsagenden zu ME/CFS wie z.B. des Instituts für Qualität und Wirtschaftlichkeit im Gesundheitswesen, das durch das Bundesministerium für Gesundheit im Februar 2021 zur Umsetzung des Beschlusses des Europäischen Parlaments zur verstärkten Unterstützung von Forschungsaktivitäten zur Erstellung eines Berichts zur Erkrankung ME/CFS aufgefordert wurde [27, 28], oder dreier Betriebskrankenkassen mit Finanzierung durch den Innovationsfonds und in Kooperation mit dem Charité Fatigue Centrum sowie ein von der niederländischen Regierung bei der nationalen HTA Agency beauftragtes Forschungsvorhaben [29] sind auf viral bedingte Fatigue fokussiert.

Für nicht postviral erkrankte Patienten mit Fatigue erhebt sich die Frage, was die Ergebnisse aus der aktuellen Forschung beispielsweise für eine tumorassoziierte Fatigue oder Fatigue bei MS bedeuten und welche Konsequenzen sich für Diagnostik, Therapie und Management dieser Patienten ergeben. Zeichnet sich ein Paradigmenwechsel ab? Muss möglicherweise stratifiziert werden nach ME, CFS, ME/CFS und SEID?

Zielführender scheint es den Autoren zu sein, vergleichende Forschung für an extremer Fatigue leidende Patienten mit unterschiedlichen Krankheitsentitäten zu betreiben [30] und Ätiologie, Pathophysiologie und mögliche Therapien interdisziplinär zu beforschen. Im Sinne des Patientenwohls und mit dem Appell für die Berücksichtigung und Beachtung oft langer Leidenskarrieren und hohem Leidensdruck sind zwingend verschiedene Fachgesellschaften zur Kooperation aufzurufen, wie es beispielsweise im onkologischen Leitlinienprogramm oder dem Ausschuss Soziales der Deutschen Diabetesgesellschaft seit langem Praxis ist. Auch für das Management von extremer Fatigue und eine symptomorientierte Therapie könnte man von der Expertise anderer Fachdisziplinen profitieren. Das gilt insbesondere hinsichtlich der Ausgestaltung von Pacing und Coping, denn für das komplexe Krankheitsbild sind Interaktionen von einzelnen Symptomen wie erschöpften psychosozialen Kompensationsmöglichkeiten (reaktive Depression), Schmerz, gestörtem Schlaf und Folgen körperlicher Inaktivität (Dekonditionierung) anzunehmen [15].
Um Patienten mit extremer Fatigue besser beraten, begleiten und adäquat an Fachärzte überweisen zu können, muss auch die Handhabbarkeit diagnostischer Werkzeuge verbessert werden. Als Vorbild können die ein- bis zweiseitigen Toolkits der amerikanischen CDC zu Beurteilung, Beschreibung, Management spezifischer Symptome und von PEM sowie weitere Strategien zum Umgang mit ME/CFS dienen [31]. Websites mit weiterführenden Informationen im Internet sind in 0 Infobox 3 aufgelistet.

\section{Schlussfolgerungen}

Patienten, die von extremer Fatigue betroffen sind, haben einen hohen Leidensdruck und werden häufig stigmatisiert. Für postinfektiöse ME/CFS gibt es viele Hinweise auf eine u.a. autoimmune Genese, die Pathophysiologie ist jedoch noch immer nicht eindeutig geklärt.

„Ich glaube das Schlimmste ist dieser Kontrollverlust und umso mehr man strampelt, dagegen ankämpft, umso schlimmer wird der Gesundheitszustand. Das ist eigentlich wie im Treibsand", beschreibt eine Betroffene ihr Leben mit ME/CFS [1]. Aktuell gibt es noch keine kausale Therapie oder Medikation. Laut Prof. Uta Behrends, deren Forschungsschwerpunkt ME/CFS bei Kindern am Klinikum rechts der Isar der TU München ist, stehen in der symptomorientierten Behandlung die Beratung, die Kreislaufstabilisierung durch Übungen ohne Belastung und die Entspannung im Vordergrund [1]. Ein bis zwei Prozent der an COVID-19 Erkrankten haben das Risiko, ME/CFS zu entwickeln [1]. Vor dem Hintergrund steigender Impfraten sollte die Forschung zu extremer Fatigue und ME/CFS jedoch nicht nur viral bedingte Erkrankungen umfassen.

Ein Therapieansatz kann nur durch die Zusammenarbeit von Patienten, Ärzten und Grundlagenforschern gefunden werden, wozu die Politik nach Aussage von Dr. Bhupesh Prusty von der JuliusMaximilians-Universität Würzburg einen wichtigen Beitrag leisten kann [1].

\section{Fazit für die Praxis}

- ME/CFS ist eine eigenständige Entität, aber extreme Fatigue als Teil von ME/CFS auch ein häufiges Symptom einer zugrunde liegenden Erkrankung wie beispielsweise einer Tumorerkrankung oder multiplen Sklerose.

- Um Patienten mit extremer Fatigue besser beraten, begleiten und überweisen zu können, muss die Handhabbarkeit diagnostischer Werkzeuge verbessert werden.

- In einer symptomorientierten Therapie lässt sich von der Expertise verschiedener Fachdisziplinen profitieren. Das gilt insbesondere für die Ausgestaltung von Pacing und Coping, denn für die komplexe Symptomatik bleiben Interaktionen der einzelnen Symptome wie erschöpfte psychosoziale Kompensationsmöglichkeiten, Schmerz, gestörter Schlaf und die Folgen körperlicher Inaktivität eine große Herausforderung.

- Patienten mit tumorassoziierter Fatigue oder Fatigue bei multipler Sklerose sollten daher nicht aus der aktuellen Forschung zu Fatigue ausgeschlossen werden.

Korrespondenzadresse

PD Dr. rer. medic. Barbara Buchberger, MPH

Robert Koch-Institut

Nordufer 20, 13353 Berlin, Deutschland

barbara.buchberger@gmail.com

\section{Einhaltung ethischer Richtlinien}

Interessenkonflikt. B. Buchberger, R. Zwierlein und V. Rohde geben an, dass kein Interessenkonflikt besteht.

Für diesen Beitrag wurden von den Autoren keine Studien an Menschen oder Tieren durchgeführt. Für die aufgeführten Studien gelten die jeweils dort angegebenen ethischen Richtlinien.

\section{Literatur}

1. Schmidt-Langels D Die rätselhafte Krankheit - Leben mit ME/CFS. ARTE TV 2021. https://www.arte. tv/de/videos/096283-000-A/die-raetselhaftekrankheit-leben-mit-me-cfs/. Zugegriffen: 28. Dez. 2021

2. https://www.bkk-dachverband.de/fileadmin/ Artikelsystem/Magazin/2021/Heft_2/BKK_02_ 2021_4.pdf.Zugegriffen:28.Dez. 2021

3. Nasserie T, Hittle M, Goodman SM (2021) Assessment of the frequency and variety of persistent symptoms among patients with COVID19 a systematic review. JAMA Netw Open 4(5):e2111417

4. Loblay R, Stewart G, Bertouch J et al (2002) Chronic fatigue syndrome-clinical practice guidelines-2002. Med J Aust 176:S17-S56

5. Carruthers BM, Jain AK, De Meirleir KL et al (2003) Myalgic encephalomyelitis / chronic fatigue syndrome: clinicalworking case definition, diagnostic and treatment protocols. J Chronic Fatigue Syndr 11(1):7-116 
6. Nacul L, Authier FJ, Scheibenbogen C et al (2021) European network on myalgic encephalomyelitis/ chronic fatigue syndrome (EUROMENE): expert consensus on the diagnosis, service provision, and care of people with ME/CFS in europe. Medicina 57:510

7. Stojanovic J, Boucher VG, Boyle J et al (2021) COVID-19 is not the flu: four graphs from four countries. Front Public Health 9:628479

8. Benaim AR, Sobel JA, Almog R et al (2021) Comparing COVID-19 and influenza presentation and trajectory. Front Med 8:656405

9. Osuchowski MF, Winkler MS, Skirecki T et al (2021) The COVID-19 puzzle: deciphering pathophysiology and phenotypes of a new disease entity. Lancet Respir Med 9:622-642

10. Theobald SJ, Simonis A, Georgomanolis T et al (2021) Long-lived macrophage reprogramming drives spike protein-mediated inflammasome activation in COVID-19. EMBO Mol Med:e14150

11. Yang AC, Kern F, Losada Petal (2021) Dysregulation of brain and choroid plexus cell types in severe COVID-19. Nature. https://doi.org/10. 1038/s41586-021-03710-0

12. Halpert G, Shoenfeld $Y$ (2020) SARS-CoV-2, the autoimmune virus. Autoimmun Rev 19(12):102695

13. Matschke J, Lütgehetmann $M$, Hagel $C$ et al (2020) Neuropathology of patients with COVID19 in Germany: a post-mortem case series. Lancet Neurol 19:919-929

14. Kedor C, Freitag H, Meyer-Arndt L et al Chronic COVID-19 Syndrome and Chronic Fatigue Syndrome (ME/CFS) following the first pandemic wave in Germany-a first analysis of a prospective observational study. https://www.medrxiv. org/content/10.1101/2021.02.06.21249256v1. Zugegriffen:28. Dez. 2021

15. Committee on the Diagnostic Criteria for Myalgic Encephalomyelitis/Chronic Fatigue Syndrome, Board on the Health of Select Populations, Institute of Medicine (2015) Beyond myalgic encephalomyelitis/chronic fatigue syndrome: redefining an illness. The national academies collection: reports funded by national institutes of health. National Academies Press, Washington (DC)

16. Jason LA, Kot B, Sunnquist M et al (2015) Chronic fatigue syndrome and myalgic encephalomyelitis: toward an empirical case definition. Health Psychol Behav Med 3(1):82-93

17. https://www.cdc.gov/me-cfs/index.html. Zugegriffen:28. Dez. 2021

18. Stussman B, Williams A, Snow J et al (2020) Characterization of post-exertional malaise in patients with myalgic encephalomyelitis/chronic fatigue syndrome. Front Neurol 11:1025 https:// doi.org/10.3389/fneur.2020.01025. eCollection 2020

19. ESMO Guidelines Committee, Fabi A, Bhargava R, Fatigoni $S$ et al (2020) Cancer-related fatigue: ESMO Clinical Practice Guidelines for diagnosis and treatment. Ann Oncol 31(6):713-723

20. https://www.nice.org.uk/guidance/cg186/ chapter/Recommendations\#ms-symptommanagement-and-rehabilitation.Zugegriffen: 28. Dez. 2021

21. Broch L, Smith Simonsen C, Øyen Flemmen $\mathrm{H}$ et al (2021) High prevalence of fatigue in contemporary patients with multiple sclerosis. Mult Scler J Exp TransIClin 7(1):1-10

22. https://www.nice.org.uk/guidance/ng206/ resources/myalgic-encephalomyelitis-orencephalopathychronic-fatigue-syndrome-

\section{Post-corona fatigue - a familiar picture in a new guise?}

Background: Myalgic encephalitis or chronic fatigue syndrome (ME/CFS) has again come into focus as a result of coronavirus disease 2019 (COVID-19). Fundamentally problematic is the fact that ME/CFS is considered a separate entity; however, extreme fatigue is also a common symptom of an underlying disease. Our article aims to increase the acceptance of ME/CFS and extreme fatigue facing a symptomatology that is not fully understood, and to highlight the need for research, orientation for physicians, and counselling services for patients.

Materials and methods: Orientative research by focused information gathering. Results: In various research projects, the hypothesis of post-infectious ME/CFS as an autoimmune disease could be confirmed. In general, the heterogeneity of diagnostic criteria as well as the variety of formulations to describe the symptomatology and different coding options make it difficult to clearly assign symptoms to a clinical picture. Exertion intolerance has been identified as a severe symptom of post-COVID19 disorder. For this reason, recommendations in international guidelines are currently being revised, especially with regard to pacing. The implications for recommendations in tumor-related fatigue or due to multiple sclerosis are unclear.

Conclusion: Against the background of a decreasing burden of disease due to increasing vaccination rates, research on fatigue should not only include viral diseases.

\section{Keywords}

Myalgic encephalitis · Chronic fatigue syndrome $\cdot$ ME/CFS · Post-exertional malaise $\cdot$ PEM $\cdot$ Stress intolerance Pacing

diagnosis-and-management-pdf-66143718094021. Zugegriffen: 14.Nov. 2021

23. Bested AC, Marshall AM (2015) Encephalomyelitis/ chronic fatigue syndrome: an evidence-based approach to diagnosis and management by clinicians. Rev Environ Health 30(4):223-249

24. Goudsmit EM, Nijs J, Jason LA et al (2012) Pacing as a strategy to improve energy management in myalgic encephalomyelitis/chronic fatigue syndrome: a consensus document. Disabil Rehabil 34(13):1140-1147

25. Hvidberg MF, Schouborg Brinth L, Olesen AV et al (2015) The health-related quality of life for patients with myalgic encephalomyelitis/chronic fatigue syndrome (ME/CFS). PLoSONE 10(7):e132421

26. https://www.mecfs.de/stellungnahme-leitliniemuedigkeit/.Zugegriffen:28. Dez. 2021

27. https://www.iqwig.de/download/n21-01_mecfs-aktueller-kenntnisstand_berichtsplan_v1-0. pdf?rev=209654.Zugegriffen: 28. Dez. 2021

28. https://www.europarl.europa.eu/doceo/ document/TA-9-2020-0140_DE.html. Zugegriffen: 11. Juli 2021

29. https://www.zonmw.nl/fileadmin/zonmw/ documenten/gehandicapten_en_chronisch_ zieken/MEcvs/20210224_Research_agenda_ MEcfs_def.pdf.Zugegriffen:28.Dez. 2021

30. Cotler J, Holtzmann C, Dudun C, Jason LA (2018) A brief questionnaire to assess post-exertional malaise. Diagnostics 8(3):66

31. https://www.cdc.gov/me-cfs/healthcareproviders/index.html. Zugegriffen: 28. Dez. 2021 